\title{
Magnitud y características de la intoxicación por alcohol metílico. Hospital Nacional Dos de Mayo
}

\author{
Carlos Contreras Camarena*1,a; Humberto Lira Veliz ${ }^{1, b}$; Katherine Contreras G ${ }^{2, c}$; Doris Gala Ad \\ RESUMEN
}

Objetivo: Determinar la magnitud, características clínicas y bioquímicas de la intoxicación por alcohol metílico en pacientes admitidos al Servicio de Emergencia del Hospital Nacional Dos de Mayo.

Materiales y métodos: Estudio analítico. La población fueron los pacientes que ingresaron a Emergencia del Hospital Nacional Dos de Mayo con diagnóstico de intoxicación por metanol (CIE 10: T51.1). Las variables fueron epidemiológicas (edad, sexo, ocupación, uso de narcóticos, cocaína, alcoholismo crónico e infección por HIV), clínicas (intoxicación por metanol) y bioquímicas (glicemia, urea, creatinina, electrolitos séricos y, especialmente, metanolemia). Se empleó el software SPSS, versión 23.00. Se determinaron medidas de tendencia central y de dispersión, y también Odds Ratio con sus respectivos intervalos de confianza al $95 \%$, se consideró estadísticamente significativo el valor de $p<$ de 0,05 .

Resultados: El $90 \%$ de casos fue ocasionado por ingesta accidental de bebida adulterada. El 87,5\% de los pacientes eran de nivel socioeconómico bajo. La metanolemia promedio fue de $72,04 \mathrm{mg} / \mathrm{L}(\mathrm{DE}+39,70)$ y la mortalidad global fue de $12,5 \%$. El perfil bioquímico predominante fue acidosis metabólica severa con anión gap elevado.

Conclusiones: La intoxicación por metanol representa el 0,15\% del total de consultas de emergencia; el perfil clínico se caracteriza por hipotensión, taquicardia, taquipnea, asociado a trastorno del sensorio, vómitos, midriasis y nistagmo, respectivamente. La acidosis metabólica severa se relacionó con incremento de morbimortalidad.

Palabras clave: Intoxicación alcohólica; Alcohol metílico; Emergencias (Fuente: DeCS BIREME).

\section{Magnitude and features of methanol poisoning. Hospital Nacional Dos de Mayo}

\section{ABSTRACT}

Objective: To determine the magnitude, and clinical and biochemical features of methanol poisoning in patients admitted to the emergency room of the Hospital Nacional Dos de Mayo.

Materials and methods: An analytical study was performed with data from patients diagnosed with methanol poisoning who were admitted to the emergency room of the Hospital Nacional Dos de Mayo, Lima, Peru. Collected data included age, gender, occupation, place of origin, cocaine use, chronic alcoholism, HIV infection, and clinical data associated with methanol poisoning. For the analysis, statistical measures of central tendency and dispersion were used, and $2 \times 2$ contingency tables were developed in the bivariate analysis. For the evaluation of the likelihood ratio, confidence intervals were established at $95 \%$, and a p-value $<0.05$ was considered significant.

Results: Ninety percent $(90 \%)$ of the cases occurred due to the accidental ingestion of an adulterated drink. Eightyseven point five percent $(87.5 \%)$ of the patients had low socioeconomic status. Average blood methanol level was 72.04 $\mathrm{mg} / \mathrm{L}(\mathrm{SD} \pm 39.70)$ and overall mortality was $12.5 \%$. The predominant biochemical profile was severe high-anion gap metabolic acidosis.

Conclusions: Methanol poisoning accounts for $0.15 \%$ of total emergency room consultations. Its clinical profile is characterized by hypotension, tachycardia, tachypnea, sensory disorders, vomiting, mydriasis and nystagmus. Severe metabolic acidosis was associated with increased morbidity and mortality.

Keywords: Alcoholic intoxication; Methanol; Emergencies (Source: MeSH NLM).

1. Hospital Nacional Dos de Mayo. Lima, Perú.

2. Universidad Ricardo Palma. Lima, Perú.

a. Médico Internista. MD. FACP, Profesor asociado UNMSM.

b. Medico Intensivista.

c. Medica Cirujana.

d. Enfermera especialista en Salud Pública y Comunitaria.

* Autor corresponsal. 


\section{INTRODUCCIÓN}

La intoxicación por metanol $\left(\mathrm{CH}_{3} \mathrm{OH}\right)$ es una entidad clínica poco frecuente en las salas de emergencia. Las intoxicaciones producidas por el metanol son consecuencia de su uso fraudulento en bebidas alcohólicas en sustitución del etanol o por deficiencias en el proceso de destilación, lo que ha dado lugar a intoxicaciones epidémicas en diferentes partes del mundo. La mayor parte de los casos ocurren en personas con alcoholismo crónico, que por su dependencia y sus escasos recursos económicos se ven obligados a consumir bebidas adulteradas de bajo costo. El consumo generalmente se produce en las calles o en locales públicos sin la debida autorización para su expendio de bebidas alcohólicas, por parte del municipio ni del Ministerio de Salud. Una proporción menor de pacientes consumen el metanol con fines suicidas o autolíticos. El metanol es un alcohol primario tóxico cuyo peso molecular es de 32 daltons, es un líquido, incoloro, volátil y soluble en agua(1). En los seres humanos la toxicidad se produce a través de sus productos metabolitos, formaldehído y, especialmente, el ácido fórmico que llega a producir disfunción neurológica, metabólica, respiratoria, renal y cardiovascular; en algunos casos se puede producir ceguera permanente y muerte. El metanol es absorbido y rápidamente distribuido en el organismo; no se une a proteínas y tiene un volumen de distribución de $0,6-1 / \mathrm{kg}$ de peso.

La mayor parte del metanol circula en el agua plasmática, atraviesa la barrera hematoencefálica y es metabolizado lentamente en el hígado. La vida media oscila entre 12 a 24 horas. Apenas cerca del $10 \%$ es excretado sin cambios por el riñón y a través del pulmón. El 90 \% restante es metabolizado a acido fórmico que se elimina por vía urinaria. Una vez absorbido se dirige al hígado donde sufre procesos de oxidación. La enzima responsable de su transformación es el alcohol deshidrogenasa que lo oxida a formaldehído y éste a su vez es oxidado a ácido fórmico por el aldehído deshidrogenasa. La acidosis sistémica es causada por el ácido fórmico y por el ácido láctico que se genera por la alteración del proceso de fosforilación oxidativa; mientras que la ceguera es causada por los efectos tóxicos del ácido fórmico. La intoxicación por metanol no es exclusividad de los pacientes alcohólicos crónicos, que consumen alcohol de dudosa procedencia, sino que, además pueden presentarse en personas que manipulan productos, solventes o sustancias que contienen el alcohol metílico. Representa el $0,3 \%$ de todas las intoxicaciones que, a su vez, puede ser accidental o con fines autolíticos ${ }^{(2)}$ y el $1,5 \%$ de las intoxicaciones por alcohol (3). A pesar del tratamiento inmediato, la mortalidad es alrededor de $20 \%$ y una proporción similar tiene daño visual residual, el retraso en la terapéutica específica incrementa la morbimortalidad ${ }^{(4,5)}$.

El objetivo del estudio fue determinar la magnitud del problema, las características clínicas y bioquímicas de la intoxicación por alcohol metílico en pacientes admitidos al Servicio de Emergencia y Shock Trauma del Hospital Nacional Dos de Mayo.

\section{MATERIALES Y MÉTODOS}

\section{Diseño y población de estudio}

Se realizó un estudio de tipo observacional, descriptivo, cuantitativo, exploratorio de corte transversal y retrospectivo. La población de estudio fueron los pacientes con que ingresaron con el diagnóstico de intoxicación por metanol (CIE: T51.1) a la Emergencia del Hospital Nacional Dos de Mayo en el periodo de estudio. Se excluyeron dos casos porque no se encontraron los registros clínicos, y cuatro casos por carecer de determinación sérica de metanol.

\section{Variables y mediciones}

Se recolectaron variables epidemiológicas que incluían edad, sexo, ocupación, uso de narcóticos, cocaína, alcoholismo crónico, infección por HIV, entre otras. La variable clínica se relaciona a la intoxicación por metanol. Las variables analíticas son glicemia, urea, creatinina, electrolitos séricos, datos gasométricos como $\mathrm{pH}, \mathrm{HCO}_{3}, \mathrm{PCO}_{2}, \mathrm{PO}_{2}$ y anión gap, valores obtenidos en el laboratorio central del Hospital Nacional Dos de Mayo. La unidad de análisis fue la historia clínica, el tiempo de revisión de cada registro clínico fue aproximadamente 20 minutos. El dosaje de metanol sérico fue uno de los criterios de inclusión en el estudio.

\section{Análisis estadístico}

Los datos recolectados fueron codificados, insertados a una base de datos y procesados estadísticamente con el programa SPSS, versión 23.00 en idioma inglés. Los resultados se expresaron como distribución de frecuencias absolutas y relativas para variables cualitativas. El promedio aritmético, desviación estándar (+1DS), moda, mediana, rango y percentiles (25 y 75), fueron las principales medidas estadísticas de tendencia central que se utilizaron para el procesamiento de las variables cuantitativas. Se elaboraron tablas de contingencia de $2 \times 2$ dentro del análisis bivariado. Para el análisis de las variables dicotómicas se determinaron Odds Ratio con sus respectivos intervalos de confianza 
al $95 \%$, se consideró estadísticamente significativo el valor de $\mathrm{p}<$ de 0,05 .

\section{Consideraciones éticas}

Se mantuvo la confidencialidad de la información obtenida de la base de datos del Servicio de Estadística e Informática del Hospital.

\section{RESULTADOS}

Se encontraron 46 casos de intoxicación por metanol durante el periodo de estudio programado (primero de enero del 2000 hasta 31 de diciembre del 2015). Fueron excluidos seis casos. La edad media de la población fue 40,08 años $(D E+11,24)$, mediana de 42,5 años, la edad mínima de 16 y la máxima de 68 años. El percentil 75 para la edad correspondía a 46,00 años.

El $75 \%$ (30/40) de los pacientes fueron de sexo masculino y el $25 \%$ (10/40) de sexo femenino, la relación masculino/ femenino fue $3: 1$, no se encontró diferencia significativa respecto al sexo según el variable sexo (P: 0,97).

El $90 \%(36 / 40)$, de los pacientes ingirieron el metanol en bebidas adulteradas de dudosa procedencia en lugares de venta informales localizados cerca de mercados, emporios, celebraciones patronales, fiestas públicas, entre otros, al que denominamos ingesta accidental. El $10 \%$ (4/40), restante utilizó el alcohol metílico con fines suicidas o autolíticos.

El $40 \%(16 / 40)$ de los pacientes eran obreros; $22,5 \%$ (9/40), ambulantes; 17,5\% (7/40), desocupados; $10 \%$ (4/40), empleados; $5 \%$ (2/40) estudiantes y, finalmente, $5 \%(2 / 40)$, amas de casa.

Se determinó la prevalencia de pacientes que fueron hospitalizados por el Servicio de Emergencia durante el periodo de estudio. La mayoría de los casos se presentaron el 2000, 2005 y 2010, con cuatro casos por cada año, y la mayor parte de ellos, durante los meses de julio y diciembre (Figura 1).

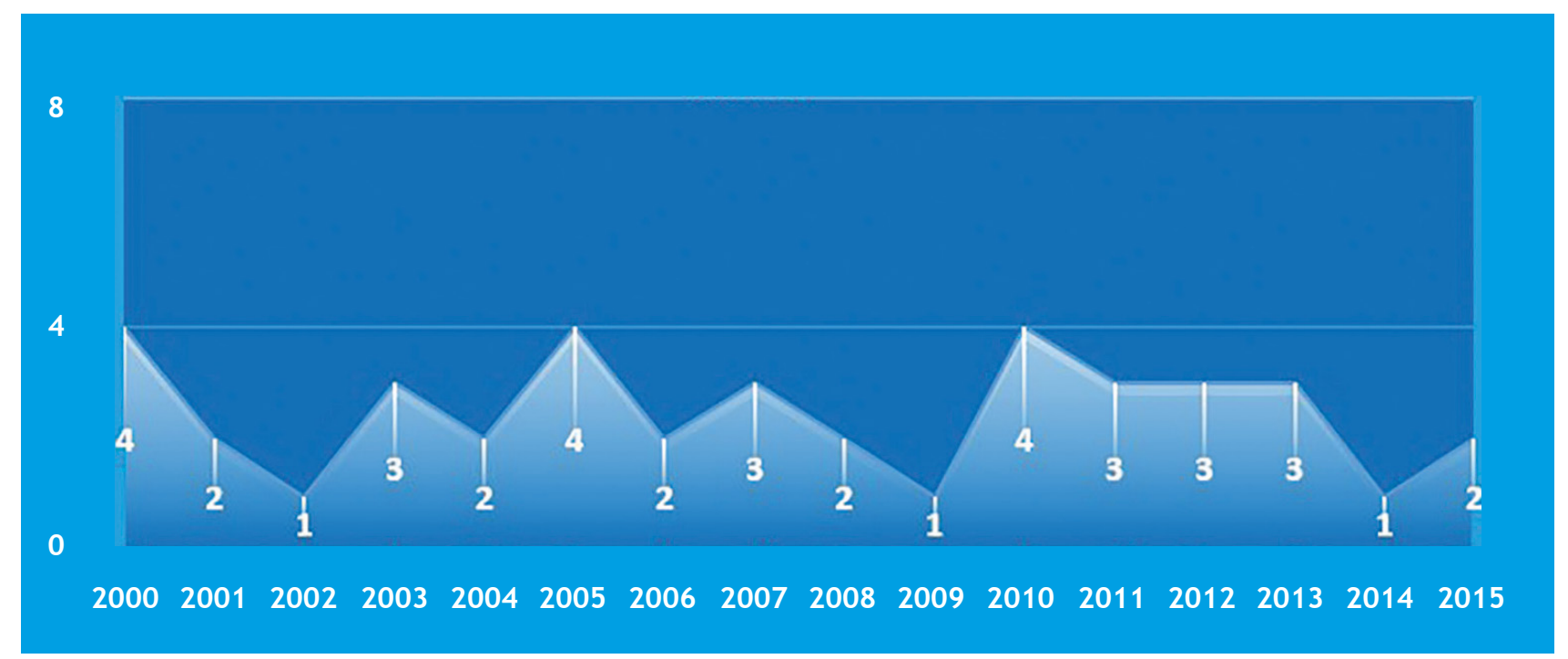

Figura 1. Intoxicación por metanol. HNDM. Lima, Perú, 2000-2015

El promedio de tiempo de hospitalización fue de 8,90 $(\mathrm{DE}+4,91)$ días, la mediana y moda fueron 7,50 y 7 respectivamente, con una estancia mínima de 1 día y máxima de 30 días.

La gran mayoría de pacientes procedieron de los distritos de La Victoria (22,5 \%), Cercado de Lima
(22,5\%), El Agustino (17,5 \%) y San Juan de Lurigancho $(10 \%)$. Otra proporción de pacientes hospitalizados venían de algunos distritos alejados de Lima, incluso lugares que no corresponden a la jurisdicción del Hospital Dos de Mayo como Ate, Comas, Carabayllo, Breña, San Bartolo, entre otros (Figura 2). 


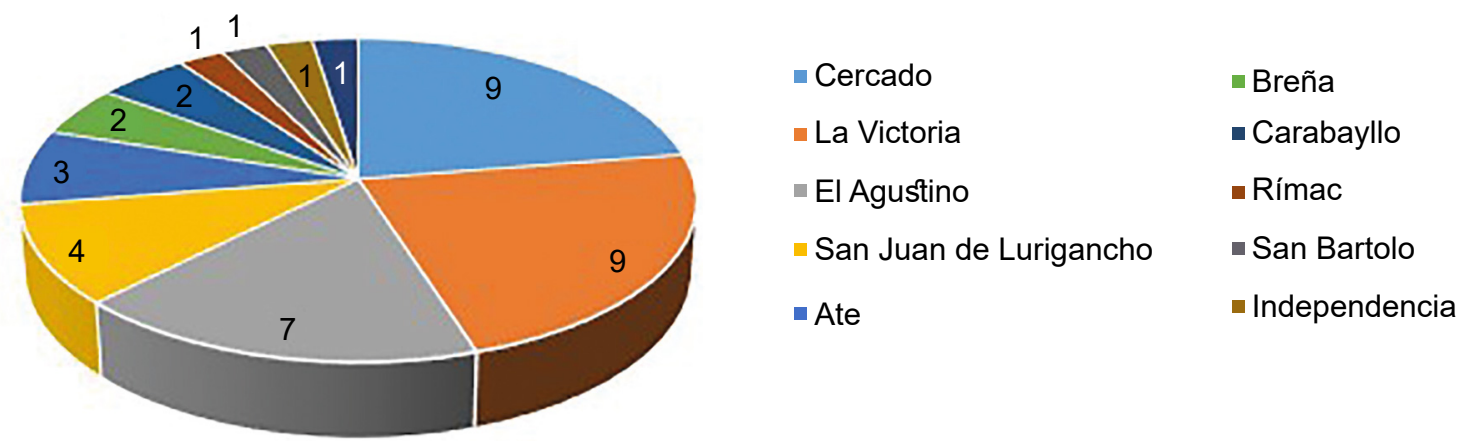

Figura 2. Procedencia de pacientes intoxicados por metanol. HNDM. Lima, Perú. 2000-2015

Respecto al grado de instrucción de los pacientes incluidos, el 42,5\% correspondían al nivel primario, $45 \%$ tenían estudios de secundaria; $10 \%$, estudios técnicos (en institutos tecnológicos o carreras técnicas), y solo el 2,5\% tenían estudios superiores universitarios.

La sintomatología presentada por los pacientes con intoxicación por metanol fue diversa y florida, con abundancia de síntomas y signos, probablemente por el estado de gravedad con que acudían a las salas de urgencia. El trastorno del sensorio (100\%), vómitos $(95,3 \%)$, visión borrosa $(82,5 \%)$, respiración acidótica $(82,5 \%)$ y nistagmo $(72,5 \%)$ fueron las alteraciones clínicas más frecuentes encontrados en la serie estudiada. Otras manifestaciones registradas con menor frecuencia, pero no menos importantes, fueron midriasis $(57,5 \%)$, cefalea $(45 \%)$, dolor abdominal $(42,5 \%)$, agitación psicomotriz (40\%), amaurosis $(25,0 \%)$, anisocoria (20\%) y convulsiones (20\%) (Tabla 1).

Tabla 1. Características clínicas en pacientes con intoxicación por alcohol metílico, HNDM. 2000-2015

\begin{tabular}{|lcc|}
\hline \multicolumn{1}{|c}{ Síntomas o signos } & Frecuencia & Porcentaje \\
\hline Alteración del sensorio & 40 & $100,00 \%$ \\
\hline Vómitos & 38 & $95,00 \%$ \\
\hline Visión borrosa & 33 & $82,50 \%$ \\
\hline Respiración acidótica & 33 & $82,50 \%$ \\
\hline Nistagmo & 29 & $72,50 \%$ \\
\hline Midriasis & 23 & $57,50 \%$ \\
\hline Cefalea & 18 & $45,00 \%$ \\
\hline Dolor abdominal & 17 & $42,50 \%$ \\
\hline Agitación psicomotriz & 16 & $40,00 \%$ \\
\hline Amaurosis & 10 & $25,00 \%$ \\
\hline Convulsiones & 8 & $20,00 \%$ \\
\hline Anisocoria & 8 & $20,00 \%$ \\
\hline
\end{tabular}

El promedio de PAS (presión arterial sistólica) fue de $95,28(\mathrm{DE}+11,03)$ y el promedio de PAD (presión arterial diastólica) fue 59,70 (DE+10,05). La media de la frecuencia cardiaca y de la frecuencia respiratoria fue de $114,13(D E+16,77)$ y $27,25(D E+5,84)$, respectivamente.
La gran mayoría de pacientes con intoxicación por metanol presentaron cifras de hipotensión leve a moderada, fue infrecuente la hipotensión severa y refractaria al tratamiento. 
Del total de los pacientes incluidos para el procesamiento de la información, 5 fallecieron y 10 presentaron secuela visual irreversible (amaurosis). Para optimizar el análisis bivariado respecto a mortalidad asociado a nivel de $\mathrm{pH}$ sérico, se incluyeron al grupo de fallecidos, los pacientes que presentaron secuela permanente (amaurosis). Luego del análisis bivariado, se encontró el test de $\mathrm{x}^{2}: 3,00$ para 1 grado de libertad. Se determinó el riesgo de morir o de sufrir secuela permanente en relación con los niveles séricos del pH. Se encontró que el grupo de pacientes con niveles de $\mathrm{pH}<$ de 6,74 tenían hasta tres veces más probabilidades (OR: 3,18 ) de fallecer y de quedar con secuela permanente (Tabla 2).

Tabla 2. Riesgo de fallecer o presentar secuela según nivel del pH

\begin{tabular}{rlllllll|}
\hline pH & \multicolumn{2}{c}{ Fallecido/secuela } & \multicolumn{2}{c}{ Vivos } & \multicolumn{2}{c}{ TOTAL } \\
& $\mathbf{N}$ & $(\%)$ & $\mathbf{N}$ & $(\%)$ & N & $(\%)$ \\
\hline$<6,74$ & 9 & 60 & 8 & 32 & 17 & 42,5 \\
\hline $\mathbf{6 , 7 4}$ & 6 & 40,0 & 17 & 68,0 & 23 & 57,5 \\
& 15 & 100,0 & 25 & 100,0 & 40 & 100,0 \\
\hline
\end{tabular}

(OR: 3,18 IC 95\%: 0,84 - 12,0)

La media del dosaje sérico de metanol fue $72,04 \mathrm{mg} / \mathrm{L}$ (DE+39,70), la mediana de $61,18 \mathrm{mg} / \mathrm{L}$, el valor mínimo y el máximo fue de $23,74 \mathrm{mg} / \mathrm{Ly} 168,00 \mathrm{mg} / \mathrm{L}$, respectivamente. El percentil 25 y 75 correspondía a $39,80 \mathrm{mg} / \mathrm{L}$ y 94,74 $\mathrm{mg} / \mathrm{L}$ respectivamente. Se encontró que el promedio de los niveles de metanolemia en esta investigación fueron superiores comparados con otros estudios publicados, lo que evidencia la correlación con la severidad del cuadro clínico.

No se encontró hallazgos anormales significativos respecto a los niveles de los electrolitos séricos. Las características bioquímicas como la glicemia, creatinina, bicarbonato, $\mathrm{pH}, \mathrm{PCO}_{2}, \mathrm{PO}_{2}$ y anión gap, entre otras, se muestran en el cuadro 3. Resalta la acidosis metabólica severa, los bajos niveles de bicarbonato y el incremento casi constante del anión gap. AG: 27,87 (DE: $\pm 6,24$ ).

De los 40 pacientes ingresados al estudio, 5 fallecieron lo que significa una mortalidad hospitalaria de $12,5 \%$. Diez pacientes ( $25 \%$ ), quedaron con secuela visual permanente, amaurosis bilateral, lo que confirma que esta es la complicación más grave e incapacitante en estos pacientes.

Tabla 3. Características bioquímicas en pacientes con intoxicación aguda por metanol HNDM. Lima, Perú. 2000-2015

\begin{tabular}{|c|c|c|c|c|c|c|c|}
\hline Estadísticos & $\begin{array}{c}\text { Metanol } \\
\text { (mg/L) }\end{array}$ & Glucemia & Urea & Creatinina & $\mathrm{HCO}_{3}$ & $\mathrm{pH}$ & $\begin{array}{c}\text { Anión } \\
\text { gap }\end{array}$ \\
\hline Media & 72,04 & 100,65 & 52,19 & 2,39 & 5,66 & 6,74 & 27,87 \\
\hline Mediana & 61,18 & 93,50 & 36,93 & 1,72 & 4,30 & 6,78 & 27,40 \\
\hline Moda & 24,65 & 60,00 & 32,00 & 1,80 & 3,60 & 6,60 & 24,44 \\
\hline D.S. ( $\pm D S)$ & 39,70 & 39,81 & 39,88 & 1,89 & 2,96 & 0,33 & 6,24 \\
\hline Rango & 144,26 & 194,00 & 166,02 & 8,66 & 11,60 & 1,23 & 30,00 \\
\hline Mínimo & 23,74 & 37,00 & 18,98 & 0,92 & 1,40 & 6,13 & 17,00 \\
\hline Máximo & 168,00 & 231,00 & 185,00 & 9,58 & 13,00 & 7,36 & 47,00 \\
\hline
\end{tabular}

DS: desviación estándar. HNDM: Hospital Nacional Dos de Mayo. 


\section{DISCUSIÓN}

La intoxicación por metanol o alcohol metílico, a pesar de su escasa frecuencia, constituye un problema de salud pública. Con frecuencia está asociado a personas que padecen de alcoholismo crónico que consumen bebidas adulteradas estimuladas por su bajo costo ${ }^{(6,7)}$. La intoxicación por alcohol metílico representa el $0,15 \%$ de todas las consultas de medicina realizadas en las salas de emergencia del Hospital Nacional Dos de Mayo, característicamente estos pacientes acuden con severo deterioro del equilibrio ácido-base, y a pesar del tratamiento precoz, muchos de ellos quedan con secuela visual o fallecen. La mayoría de los pacientes que presentan intoxicación por metanol tienen una escasa escolaridad y bajo nivel educativo, más del $80 \%$ de los casos solo tuvieron estudios primarios y secundarios incompletos.

Nuestro estudio de 15 años nos ha permitido determinar la prevalencia de la intoxicación por metanol, la frecuencia es de 1 a 4 casos por año, sin información de intoxicaciones masivas en nuestro medio, como las descritas en Colombia ${ }^{(8)}$, Ecuador, Guatemala, Nicaragua, República Checa (9) y Estados Unidos de Norteamérica. En el Perú, la mayoría de las publicaciones, son de reportes de casos clínicos o serie de casos con complicaciones graves como hemorragias e isquemias cerebrales, especialmente, a nivel de los ganglios basales ${ }^{(10,11)}$.

La intoxicación por metanol ocurre con mayor frecuencia en varones, sobre todo en la tercera y cuarta década de la vida, tal como se informa en la mayoría de las investigaciones ${ }^{(8,9,11)}$. En nuestra serie la edad promedio fue de 40,08 (DE+11,24). La proporción encontrada respecto al sexo masculino en relación al femenino fue de $3: 1$, esta predominancia del género masculino fue reportada en diferentes series de otros países ${ }^{(12-14)}$, esta característica podría estar relacionada a la mayor proporción de varones con dependencia alcohólica y su contacto más frecuente en sus actividades laborales con productos tóxicos industriales que contienen metanol.

La mayoría de los casos de intoxicación fueron por ingesta accidental de bebidas alcohólicas adulteradas de bajo costo, en personas con alcoholismo crónico, que estuvieron bebiendo licor durante 24 a 48 horas previas a su ingreso por emergencia. Cuatro de los pacientes de la serie, tres de ellos mujeres, tuvieron antecedentes psiquiátricos, consumo de cocaína y terapia con benzodiacepinas. Estos pacientes bebieron alcohol adulterado, pero no accidentalmente sino con fines suicidas, es decir, sabiendo que el alcohol era dañino decidieron beber con fines autolíticos.

El $72,5 \%$ de los pacientes proceden de distritos contiguos que pertenecen a la jurisdicción del hospital. Debido a su ubicación céntrica, el Hospital Dos de Mayo se encuentra rodeado de distritos populosos, emporios, mercados populares, donde abundan los trabajadores informales, ambulantes, venta de objetos usados en mercados insalubres, obviamente, no faltan cantinas o "chinganas", lugares donde se expenden licores a bajo costo, muchas veces preparados en forma artesanal y empírica, condiciones que favorecen la ingesta de licor adulterado en personas de bajos recursos y con dependencia alcohólica. El Hospital Dos de Mayo es un hospital docente de tercer nivel y referente nacional, que por su ubicación se ve favorecido con la admisión de estos pacientes. El 87,5 \% de los pacientes solo tenían estudios primarios $\mathrm{y} / \mathrm{o}$ secundarios incompletos, el $40 \%$ de ellos eran ambulantes y desocupados, constituyendo un grupo de vulnerable de morir por la toxicidad del alcohol metílico, debido a la presencia de comorbilidades.

Este hallazgo contrasta con estudios realizados en otros países en donde el nivel socioeducativo es diferente y predominan ocupaciones como pescadores, marineros, barnizadores, carpinteros, entre otros ${ }^{(8,12-14)}$.

El $100 \%$ de los pacientes tuvieron trastorno del sensorio en diferentes grados: somnolencia (50\%), sopor $(25 \%)$, estupor $(10 \%)$, y coma $(15 \%)$, al parecer los diferentes aspectos metabólicos del metanol que actúan aditivamente hace que ningún paciente se libre de presentar trastorno del sensorio aun en su grado más leve (somnolencia). Estos hallazgos concuerdan con los encontrados por Paasma et al., en su estudio multicéntrico, donde el compromiso de conciencia (escala de Glasgow menor a 8), estuvo presente en $30 \%$ del total de pacientes de su serie, y en el $85,4 \%$ en el grupo que fallecieron ${ }^{(14)}$.

Los vómitos $(95,3 \%)$, visión borrosa $(82,5 \%)$, respiración acidótica $(82,5 \%)$, nistagmo $(72,50 \%)$ y midriasis $(57,50 \%)$ fueron las otras alteraciones que, frecuentemente, acompañaron a la mayoría de los pacientes. Es importante sospechar de intoxicación por metanol en todo paciente adulto joven generalmente con dependencia de alcohol con bajo nivel educativo, que tiene antecedente de ingesta de bebidas alcohólicas baratas y acude a la emergencia con vómitos, visión borrosa, respiración acidótica y con algún grado de alteración del sensorio y que, adicionalmente, al examen físico presenta midriasis 
areactiva y nistagmo.

Una vez ingerido, el metanol se absorbe a nivel gastrointestinal y alcanza su pico máximo a los 30 90 minutos, el $20 \%$ se elimina por vía pulmonar y renal sin metabolizarse, el $80 \%$ se metaboliza a nivel hepático por la enzima alcohol deshidrogenasa que origina formaldehído y este, por acción de la aldehído deshidrogenasa, pasa a ácido fórmico, este último; es el responsable de la toxicidad, pues no solo genera acidosis metabólica, sino que, además, bloquea la respiración celular al bloquear la citocromo oxidasa mitocondrial y ocasionar daño citotóxico, metabolismo anaeróbico y producción de acidosis láctica ${ }^{(15,16)}$.

El paciente con intoxicación por metanol tiene alteración de las constantes clínicas caracterizada especialmente por hipotensión, tanto sistólica como diastólica, que en algunos pacientes llegaron al shock refractario. Este hallazgo concuerda con los encontrados en otras investigaciones realizadas en Perú (11), y en otros países. Varona et al. (17), reporta 5 pacientes en el hospital de Basurto, Bilbao, el $100 \%$ de los casos, presentó shock al momento de acudir a la emergencia, igualmente, Blas et al. ${ }^{(18)}$, reportó un caso de intoxicación por metanol que fue admitido al hospital SSA de Durango, México con PA: 70/40. Algunas series reportan pacientes con PA normal o discretamente baja ${ }^{(19,20)}$, el promedio encontrado en nuestra serie fue de PAS (presión arterial sistólica), 95,28 $(\mathrm{DE}+11,03)$ y PAD (presión arterial diastólica), 59,70 (DE+10,05). No solo se altera la PA si no también la frecuencia cardiaca y respiratoria que se incrementan en la mayoría de los pacientes.

Los niveles de metanol sérico mayores que $\mathrm{mg} / \mathrm{L}$ se consideran tóxicos y mayores a $40 \mathrm{mg} / \mathrm{L}$, mortales; existen diferentes investigaciones que tratan de relacionar niveles séricos elevados de metanol con incremento de mortalidad (20,21). Contrariamente, se han reportado casos no fatales de pacientes intoxicados con metanol sérico de hasta $350 \mathrm{mg} / \mathrm{L}$; y otros, con niveles alrededor de $40 \mathrm{mg} / \mathrm{L}$, que fueron mortales ${ }^{(22)}$. Estos datos contradictorios en la literatura obligan a realizar nuevas investigaciones. El promedio del dosaje sérico de metanol encontrado en nuestra investigación fue $72,04 \mathrm{mg} / \mathrm{L}(\mathrm{DE}+39,70)$, la mediana de $61,18 \mathrm{mg} / \mathrm{L}$, el valor mínimo y el máximo fue de $23,74 \mathrm{mg} / \mathrm{L}$ y $168 \mathrm{mg} / \mathrm{L}$, respectivamente. El percentil 25 y 75 correspondía a $39,80 \mathrm{mg} / \mathrm{L}$ y 94,74 $\mathrm{mg} / \mathrm{L}$, respectivamente. Los pacientes que fallecieron tenían concentraciones séricas de metanol de 96,75, $28,24,149,00,57,00$ y $82,85 \mathrm{mg} / \mathrm{L}$. El promedio de metanol sérico en este grupo de pacientes fue 82,76 $(\mathrm{DE}+45,32)$. El $80 \%$ de los pacientes que fallecieron tenían niveles de metanol séricos por encima de 50 $\mathrm{mg} / \mathrm{L}$. Los pacientes con niveles de $\mathrm{pH}<6,74$ tuvieron hasta tres veces más probabilidades de fallecer o de quedar con ceguera permanente e irreversible (OR: 3,18 ), resultados concordantes con los estudios de diferentes investigaciones ${ }^{(14,20-22)}$. El tratamiento en el $100 \%$ de los casos fue con el antídoto alcohol etílico, no se usó fomepizol, por no contar con este fármaco. El $70 \%$ de los pacientes (28/40), necesitaron apoyo de hemodiálisis, especialmente aquellos pacientes que tenían niveles de metanolemia superior a $50 \mathrm{mg} / \mathrm{L}$, con acidosis severa y con algún trastorno visual, siguiendo las recomendaciones de las guías de tratamiento ampliamente difundidas en diferentes partes del mundo ${ }^{(23-25)}$.

La tasa de mortalidad hospitalaria en nuestro estudio fue del $12,5 \%$ cifra muy inferior a la reportadas en diferentes estudios donde se describen mortalidad alrededor del 18 al $24 \%$ en intoxicaciones masivas en Noruega y Estonia ${ }^{26,27)}$. La severidad clínica de los pacientes incluidos en nuestra investigación, fueron similares a los pacientes intoxicados masivamente en los países descritos anteriormente.

A pesar del tratamiento especializado y oportuno en las salas de emergencia y cuidados intensivos, una proporción de pacientes ( $25 \%$ ), quedaron con secuela visual permanente.

Resultado mucho mayor al encontrado por Paasma et al., quien reporta $17,5 \%$ de secuela irreversible. La presencia de acidosis metabólica severa y los niveles de metanol sérico superiores a $50 \mathrm{mg} / \mathrm{L}$ tienden a incrementar morbimortalidad y complicaciones hemorrágicas en pacientes intoxicados con alcohol metílico $^{(14,17,24,28,29)}$.

En conclusión, la intoxicación por metanol representa el $0,15 \%$ del total de consultas de emergencia; el perfil clínico se caracteriza principalmente por hipotensión, taquicardia, taquipnea, asociados a trastorno del sensorio, vómitos, midriasis y nistagmo, respectivamente. La acidosis metabólica severa $(\mathrm{pH}<6,74)$ se relacionó con incremento de morbimortalidad.

\section{REFERENCIAS BIBLIOGRÁFICAS}

1. Sharma A, Gold Frank's Toxicologic Emergencies 7th Ed. New York: McGraw-Hill; 2002:980-1001.

2. Abramson A, Singh S. Treatment of alcohol intoxications: ethylene glycol, methanol and isopropanol. Nephrology and Hypertension. 2000; 9(6):695-701. 
3. Oscanoa PE, Sierra LM, Miyahira J. Características clínicas y evolución de los pacientes con intoxicación por metanol atendidos en un hospital general. Rev Med Hered. 2010; 21(2):70-6.

4. Marruecos-Sant L. Tratamiento de las intoxicaciones por methanol y etilenglicol. Med intensiva. 2002; 26(5):248-250.

5. kuteifan K, Oesterle H, Tajahmady T, Gutbub AM, Laplattte G. Necrosis and hemorrhage of the putamen in methanol poisoning shown on MRI. Neuroradiology. 1988; 40(3):158-60.

6. Nesime $\mathrm{Y}$, Agritmis $\mathrm{H}$, Turla A, Koc S. Fatalities due to methyl alcohol intoxication in turkey: an 8 year of study. Forensic Sci Int.2003; 131(1):36-41.

7. Balaguer J. Intoxicación por Metanol. En. Toxicología Clínica. Editorial Bataller S. Valencia. 2004:60-64.

8. Varona M, Suarez G, Velasquez M, Morales P, Sanchez C. Estudio de un brote de intoxicación por alcohol metílico en Santa Fe de Bogotá. Biomédica. 1997;17(2):130-136.

9. Zakharov S, Pelclova D, Urban P, Navratil T, Diblik P, Kuthan $P$, et al. Czech mass methanol outbreak 2012: epidemiology, challenges and clinical features. Clin Toxicol. 2014;52(10): 1013-24.

10. Rojas Vera J. Lesiones hemorrágicas cerebrales en intoxicación por metanol. Med Intensiva. 2003;27(6):426-429.

11. Valdivia-Infantas M, Medina-Santander B, Arana-Retamozo R, Llamoca-Palomino V, Vásquez-Gil A. Intoxicación por metanol con hemorragia e isquemia de los ganglios basales: reporte de dos casos. Rev Soc Perú Med Interna. 2013;26(1):26-31.

12. Palacio Pérez H, Hernández Ruiz A, Delgado Fernández RI. Intoxicación por alcohol metílico: a propósito de un caso. Rev Cienc Méd. La Habana. 2013 19(3):397-401

13. Epker JL, Bakker J. Accidental methanol ingestion: Case report. BMC Emerg Med. 2010; 10(3):102-106

14. Paasma R, Hovda KE, Hassanian-Moghaddam $\mathrm{H}$, Brahmi $\mathrm{N}$, Afshari R, Sandvik L, et al. Risk factors related to poor outcome after methanol poisoning and the relation between outcome and antidotes - a multicenter study. Clin Toxicol. 2012; 50(9):823-83.

15. Córdova D. Toxicología. 4th Ed. Mexico: Editorial Manual Moderno.2000:393-398.

16. McCoy HG, Cipolle RG, Ehlers SM, Sawchuk RJ, Zaske DE. Severe methanol poisoning: application of a pharmacokinetic model by ethanol therapy and hemodialysis. Am J Med. 1979; 67(5):804807.

17. Varona Peinador M, Sanz Prieto JC, García Cuevas M, Gutiérrez Macías A, Aguirre Herrero J, Ugalde García F, et al. Intoxicación mortal por metanol. Emergencias. 1999; 11:315-319.

18. García Vicente E, García y García EL. Intoxicación aguda por metanol en un varón de 32 años. Medicrit. 2009:6(1):62-5.

19. Blas J. Nava S. Intoxicación por metanol inhalado 2000. Rev Asoc Mex Med Crit y Ter Int. 2000;14(2):67-70.

20. Sutton TL, Foster RL, Liner SR. Acute methanol ingestion. Pediatr Emerg Care. 2002; 18(5):360-3.

21. Kruse JA. Methanol poisoning. Intensive Care Med. 1992; 18(7):391-397.

22. Olivier N, Vieitiez J. Amaurosis bilateral como causa de intoxicación por metanol: a propósito de un caso. Cuad Med Forense. 2003; 32:43-47.

23. Barceloux DG, Bond GR, Krenzelok EP, Cooper H, Vale JA. American Academy of Clinical Toxicology practice guidelines on the treatment of methanol poisoning. J Toxicol Clin. 2002; 40(4): 415-446.

24. Kraut JA, Kurtz I. Toxic alcohol ingestions: clinical features, diagnosis, and management. Clin J Am Soc Nephrol. 2008; 3(1):208-225.

25. Megarbane B, Borron SW, Baud FJ. Current recommendations for treatment of severe toxic alcohol poisonings. Intensive Care Med. 2005; 31(2):189-195.

26. Hovda KE, Hunderi OH, Tafjord AB, Dunlop O, Rudberg N, Jacobsen D. Methanol outbreak in Norway 2002-2004: epidemiology, clinical features and prognostic signs. J Intern Med. 2005; 258(2):181-190.

27. Paasma R, Hovda KE, Tikkerberi A, Jacobsen D. Methanol mass poisoning in Estonia: outbreak in 154 patients. Clin Toxicol. 2007; 45(2):152-157.

28. Manuchehri AA, Alijanpour E, Daghmechi M, Ghaeminan $\mathrm{N}$, Hasan Abedi S, Nikbakhsh $\mathrm{N}$, et al . A case of methanol poisoning leading to prolonged respirator dependency with consequent blindness and irreversible brain damage. Caspian J Intern Med. 2015; 6(3):180-183

29. Vyas S, Kaur N, Sharma N, Singh P, Khandelwal N. Methanol poisoning. Neurol India. 2009;57(6): 831-6.

Fuentes de financiamiento:

Este artículo ha sido financiado por los autores.

Conflictos de interés:

Los autores declaran no tener ningún conflicto de interés.

\section{Correspondencia:}

Carlos Contreras Camarena

Dirección: Av. Colonial 3046, A-403. Lima, Perú.

Teléfono: 945933754

Correo electrónico: ccontrerashndm@hotmail.com

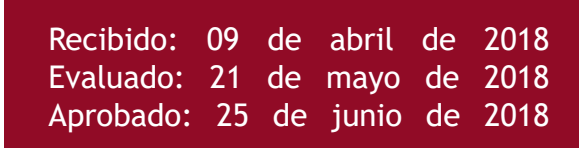

(c) La revista. Publicado por Universidad de San Martín de Porres, Perú. (c) в bajo términos de Licencia Creative Commons Atribución 4.0 Internacional. (http: //creativecommons.org/licenses/by/4.0/)

ORCID iDs

Carlos Contreras Camarena

Humberto Lira Veliz

Katherine Contreras G

Doris Gala A
https://orcid.org/0000-002-7394-995X https://orcid.org/0000-003-0634-9927 https://orcid.org/0000-003-2140-6475 https://orcid.org/0000-002-0950-8949 